\title{
Violación de derechos por discriminación de género
}

Elsa Ramos

eramos@utec.edu.sv

Investigadora UTEC

\section{Resumen}

En éste artículo se reflejan algunos los resultados de la investigación "Violación de derechos por discriminación de género", la cual indagó la violación de algunos derechos específicos de la mujer entre ellos el derecho a la salud, educación, trabajo, sexuales y reproductivos, así como el conocimiento que tienen las mujeres salvadoreñas sobre los derechos humanos y los derechos específicos de la mujer.

Palabras clave: género, discriminación, derechos específicos de la mujer, derechos humanos, violación de derechos.

\section{ABSTRACT}

This article shows some research results "Violation of rights by gender discrimination" which investigated the violation of specific rights of women including the right to health, education, employment, sexual and reproductive well as the knowledge that Salvadoran women have about human rights and specific rights of women.

Keyword: gender discrimination, gender-specific rights, human rights, violation of rights.

\section{Introducción}

En El Salvador durante los últimos años de la guerra civil (1980 -1992), cuando empiezan a surgir las primeras organizaciones de mujeres, las cuales introducen en el país de forma organizada y planificada el tema de género dentro de la problemática nacional, son éstas organizaciones de la sociedad civil con la ayuda internacional y algunas institu- ciones académicas que se dan a la tarea de realizar las primeras investigaciones relativas a la problemática específica de la mujer salvadoreña.

Al inicio de la investigación se plantearon como objetivos: analizar, determinar y cuantificar los derechos de la mujer más violentados en el país y analizar las causas que generan la violación de esos derechos, pero a medida que se avanzó 
en la lectura y análisis de la bibliografía referida al tema, se comprendió que en éste país todos los derechos de las mujeres son violentados y que el más estudiado es la violencia en sus diversas expresiones incluyendo el feminicidio (2010, ISDEMU-PNUD), por ello se optó por investigar que medida están siendo violados el derecho a la salud, educación, trabajo, la libertad de decidir sobre su propio cuerpo y los derechos sexuales y reproductivos.

\section{Método}

El método utilizado fue el mixto (Hernández, Fernández y Baptista, 2006), con una muestra probabilística por conglomerados, representativa de cada uno de los 14 departamentos del país, con una estimación de error del 3\% y un nivel de confianza del 95\% obteniéndose una muestra de 1.130 mujeres a escala nacional.

Para obtener la muestra se procedió a consultar el VI Censo de Población y $\vee$ de Vivienda realizados el año 2007 por el Ministerio de Economía, a través de la Dirección de Estadística y Censos (DIGESTYC), para obtener los datos estadísticos de las mujeres desde los 18 hasta los 50 años de edad, de los catorce departamentos que conforman la República de El Salvador.

Se utilizó un cuestionario de elaboración propia de 42 preguntas entre cerradas y abiertas, que en etapa previa fue validado a través de una prueba piloto. Se realizaron 30 entrevistas semi estructuradas a mujeres de diferentes edades, grupos sociales y nivel educativo, se entrevistó a una persona especialista en el reclutamiento de recurso humano y por último, durante el desarrollo del trabajo de campo, se realizaron mini entrevistas a algunas de las mujeres encuestadas y observación de los comportamientos y reaccio- nes de éstas ante las preguntas realizadas que ayudaron a enriquecer los resultados de la investigación.

Se integró un equipo de mujeres para la realización del trabajo de campo, el cual fue sensibilizado acerca de la violación de los derechos humanos y especiales de la mujer, se les capacitó para el llenado de las encuestas y se realizó un ensayo entre ellas mismas para calcular el tiempo aproximado que tardarían en llenar cada una de éstas. Los recursos económicos y logísticos se coordinaron con las dependencias respectivas de la Universidad Tecnológica de El Salvador. Se trabajó los fines de semana, con el objetivo de captar una muestra que incluyera tanto a mujeres residentes en las cabeceras departamentales como en los diferentes municipios, lográndose obtener encuestas de 109 municipios de los 262 existentes en el país. Los horarios de trabajo se iniciaban entre las 05:00 06:00 de la mañana y concluían entre las 06:00 y =7:00 de la noche.

Género: es la construcción social que se le asigna a mujeres y hombres con base en su sexo biológico, una identidad, roles, deberes y derechos diferenciados. También este concepto se refiere a la relación de poder entre ellos, porque sobre la base de dicha diferenciación se realiza una desigual asignación y control de los recursos materiales (dinero, tierra, vivienda, etc.) como no materiales (tiempo, ideología, instituciones, normas sociales, símbolos) entre ambos, colocando a las mujeres en una situación desventajosa frente a Ios hombres. (ISDEMU-PNUD, 2010).

Discriminación de género: de acuerdo al artículo 1 de la convención sobre la eliminación de todas las formas de discriminación contra la mujer (CEDAW por sus siglas en inglés) la expresión discriminación contra la mujer, denota toda distinción, exclusión o restricción basada en el sexo que tenga 
por objeto o por resultado menoscabar o anular el reconocimiento, goce o ejercicio por la mujer, independientemente de su estado civil, sobre la base de la igualdad del hombre y la mujer, de los derechos humanos y las libertades fundamentales en las esferas política, económica, social, cultural y civil o en cualquier otra esfera. (CEDAW, 2006).
Machismo: Hecho, costumbre o fenómeno social y cultural, que resalta la hombría y la prepotencia de los hombres sobre las mujeres en todos los campos de la vida social, laboral y afectiva. (UNESCO-ISDEMU, 2002).

\section{Derechos de la mujer (CEDAW)}

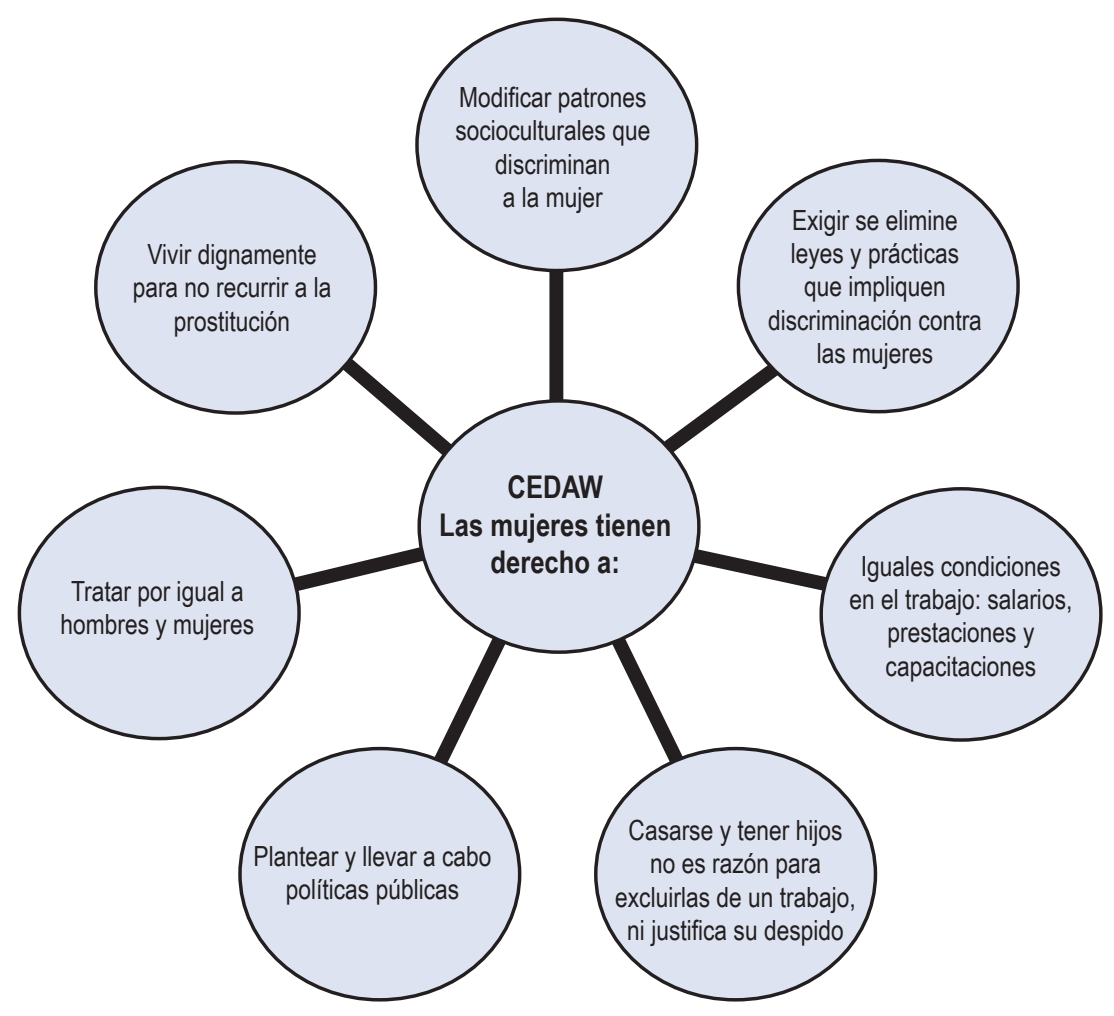

Fuente: Diagrama de elaboración propia en base a la Convención sobre la eliminación de todas las formas de discriminación de la mujer (CEDAW, 2006).

\section{Resultados}

Como resultado de los datos generales se encontró que el estado civil de las mujeres encuestadas era el siguiente: solteras 48.9\%; casadas 22.9\%; acompañadas: 19.3\%; divorciadas: $1.2 \%$; separadas: $5.2 \%$ y viudas: $2.5 \%$. en relación a la ocupación u oficio los datos obtenidos son los siguientes: estudiantes: 19.8\%; estudia y trabaja: 10.4\%; vendedoras:
32.6\%; trabajadoras domésticas: 7.5\%; pequeñas comerciantes: 4.7\%; amas de casa: 10.17\%; empleadas: $9.2 \%$; empresarias: $0.6 \%$; oficios varios: $12.2 \%$ y no contestaron: $1.4 \%$.

A continuación se exponen algunos de los resultados más relevantes obtenidos en la investigación: respecto al conocimiento que las mujeres encuestadas tenían en relación a los 
derechos humanos y los derechos específicos de la mujer, se encontró que el $60.1 \%$ de las mujeres conocían los derechos humanos, pero al revisar las respuestas de las preguntas abiertas en relación a que derechos humanos conocían, 49 de ellas manifestó que no se acordaban, cuestión que arroja que sólo un $55.75 \%$ conoce realmente los derechos humanos. Con respecto al conocimiento de los derechos específicos de la mujer, los resultados obtenidos indican que sólo un 39.6\% afirmó que los conocía, pero al pasar de igual forma a revisar las respuestas abiertas de cuales son los que conocían, 44 de ellas expresaron no recordarse de estos, igual que en el caso de los derechos humanos, disminuye el porcentaje real que conoce los derechos específicos de la mujer, quedando el porcentaje en $35.6 \%$.

Entre los derechos más violentados a las mujeres en el país se encuentra que un $42.7 \%$ de mujeres manifestaron conocer casos en donde a los hombre se les pagaba más que a las mujeres aunque realizaran el mismo trabajo, lo anterior es una violación al principio de igualdad establecido en la Constitución de la República de El Salvador en el artículo 3; al artículo 38 en su numeral 1, se establece que a igual trabajo debe corresponder igual remuneración cualquiera que sea el sexo, la raza, credo o nacionalidad de la persona trabajadora, se viola también la CEDAW, al discriminar a las mujeres por razones de género.

Un 40.2\% manifestó que conocían de casos en donde aunque las mujeres tuvieran la misma capacitación y experiencia laboral, se prefería conceder a los hombres ascensos laborales; se observa la discriminación que sufren las mujeres por razones de género. También los resultados arrojaron que un $44.9 \%$ de las encuestadas expresaron que a las mujeres para acceder a un puesto laboral se les exige la prueba de embarazo (aún cuando la ley lo prohíbe), éste último es una violación a los derechos reproductivos de la mujer, pues son las únicas que pueden dar a luz.

Al obtener los resultados del trabajo de campo se constató en general que no hay suficiente conocimiento de los derechos humanos y especiales de la mujer de acuerdo al lugar de residencia, por ejemplo, las mujeres del área urbana el $46.1 \%$ conocen los derechos humanos y sólo el $13.8 \%$ en la rural; en cuanto a los derechos especiales de la mujer, las mujeres del área urbana los conocen en un 30.5\% y las de la rural sólo el 9.2\%. Los porcentajes anteriores se repiten con uno o dos puntos porcentuales de diferencia en todos los ítems aplicados.

Se detectó que las mujeres de los departamentos de San Salvador, La Libertad, Santa Ana y San Miguel, destacan entre las que tienen mayor conocimiento de la violación a los derechos humanos y a los derechos específicos de la mujer.

En cuanto al nivel educativo de las mujeres, los resultados arrojaron que entre mayor es el número de años estudiados mayor es el conocimiento de los derechos humanos y específicos de la mujer y mayor apertura a la realización de cualquier tipo de aborto.

Hay una gran apertura por parte de las mujeres $86.9 \%$, a que se realice una amplia campaña de capacitación sobre métodos de planificación familiar, como alternativa para prevenir los embarazos de las adolescentes y también para evitar que se sigan produciendo abortos de forma clandestina (las percepciones se recogieron de los comentarios de las mujeres encuestadas). 
A partir de las respuestas abiertas acerca de por qué las encuestadas no están de acuerdo con el aborto; se detectó que las mujeres se aferran al discurso religioso y condenan abiertamente el aborto; pero al realizar mini entrevistas se dejó entrever que una cantidad considerable de ellas tienen conocimiento de cómo inducir un aborto.

\section{CONCLUSIONES}

- La mayoría de las mujeres encuestadas (55.75\%), conoce los Derechos Humanos, pero no de forma amplia.

- Los derechos especiales de la mujer sólo los conoce un $35.66 \%$.

- El desconocimiento profundo de los derechos humanos y los derechos de la mujer, conlleva a que estas mujeres no puedan acudir a las instancias respectivas para que sus derechos sean respetados.

- Se observa en el país la práctica de expulsar a mujeres adolescentes (33.3\%) de los centros educativos al estar embarazadas.

- Hacen falta más programas de capacitación para la superación de la mujer (21.9\%) y de equidad de género (17.0\%).

- $\quad$ Persiste en el país la práctica de despedir a las mujeres a causa del embarazo (26.5\%).

- Muchas empresas (44.9\%) exigen la prueba de embarazo, para dar empleo a las mujeres.
- Es práctica común exigir que la edad promedio para aceptar a una trabajadora este en el rango de los 18 a los 35 años de edad (basta revisar los anuncios de clasificados de los periódicos).

- Se continúa prefiriendo a los hombres para conceder ascensos laborales (40.2\%).

- Algunas empresas le pagan más a los hombres (42.7\%) que a las mujeres por el mismo trabajo desempeñado.

\section{REFERENCIAS}

- Constitución de la República de El Salvador. En: NORMAS BÁSICAS SOBRE DERECHOS HUMANOS. Naciones Unidas. Oficina del Alto Comisionados para los Derechos Humanos, 2000.

- Convención sobre la eliminación de todas las formas de discriminación contra la mujer (CEDAW, por sus siglas en inglés), (2006). Las Mélidas, Brot für die Welt.

- $\quad$ ISDEMU-PNUD (2010). Investigando para la igualdad: Cómo hacer de la investigación una herramienta de cambio social. San Salvador.

- $\quad$ ISDEMU-UNESCO (2006). Palabras nuevas para un mundo nuevo. San Salvador.

- $\quad$ VI Censo de población y V de Vivienda. Cifras oficiales. Población, Vivienda, Hogares. El Salvador, (2008). Ministerio de Economía. Dirección General de Estadística y Censos. Consultado en: http://www.digestyc.gob.sv/ 